\title{
LAS POLÍTICAS CULTURALES DE LOS GOBIERNOS LOCALES EN LA ARGENTINA
}

\author{
Pablo Mendes Calado \\ politicasculturales@untref.edu.ar \\ mendescalado@yahoo.com.ar \\ Instituto de Políticas Culturales Prof. Patricio Lóizaga \\ Universidad Nacional de Tres de Febrero, Argentina
}

\section{RESUMEN}

Este artículo tiene por objeto presentar el trabajo que desde el proyecto de investigación Políticas Culturales en los Gobiernos Locales ${ }^{*}$, la Universidad Nacional de Tres de Febrero viene desarrollando en este campo poco explorado del quehacer público en cultura. El trabajo en cuestión consiste en un estudio de casos mediante herramientas metodológicas tales como entrevistas en profundidad, observaciones, grupos focales, análisis presupuestario y observaciones participantes. Desde el 2007 a la fecha, doce municipios han sido objeto de estudio en Argentina. Lo que aquí se presenta son reflexiones en torno a algunos de los resultados hasta ahora obtenidos, los cuales se organizan en los siguientes temas: el desarrollo institucional, los objetivos, la nominación del destinatario y los recursos disponibles. Finalmente, se hace un balance de la actual situación que los gobiernos locales en la Argentina presentan en materia de políticas culturales y cuáles podrían ser algunos caminos para avanzar en un sentido que haga de las políticas culturales locales una fuente de desarrollo y de construcción de lazos sociales.

Palabras clave: políticas culturales, gestión cultural, cultura local, políticas culturales locales.

\footnotetext{
*Director del Proyecto: Lic. José Alejandro Tasat. Equipo de investigación: Lic. Marcela Rebón, Lic. Lorena Fiore, Lic. Fabiana Demarco, Lic. José Basualdo, Lic. Silvano Martínez, Leandro González y Lic. Pablo Mendes Calado.
} 


\section{ABSTRACT}

The objective of this article is to present the work that the Argentine National University of Tres de Febrero has been developing in the practically unexplored field of public engagement with culture, based on a research project on Cultural Policies in Local Governments*. This piece of research consists of a study of cases using various methodological tools, such as in-depth interviews, observations, focal groups, budget analysis and participant observations. From 2007 to the present, twelve municipalities have been the object of study in Argentina. This article presents reflections about some of the results obtained so far, which are organized under the following themes: institutional development, objectives, nomination of target groups, and resource availability. Finally, a balance is made regarding the current situation that local governments in Argentina present in terms of cultural policies and which could be some pathways to follow in order to turn local cultural policies into a source of development and a means to construct social bonds.

Key words: cultural policies, cultural management, local culture, local cultural policies.

\footnotetext{
*Project Director: José Alejandro Tasat, Research Team: Marcela Rebón, Lorena Fiore, Fabiana Demarco,
} José Basualdo, Silvano Martínez, Leandro González and Pablo Mendes Calado. 


\section{INTRODUCCIÓN}

En el último medio siglo las políticas cultuales han tenido importantes avances tanto en términos de praxis política, como conceptuales. Aunque desde la academia se ha generado una voluminosa bibliografía especializada, el quehacer cultural de los gobiernos locales puede decirse que es un recién llegado a este campo. Las políticas públicas en cultura que despliegan los gobiernos locales han sido un objeto de estudio escasamente visitado y su escaso desarrollo debe reconocerse en el esfuerzo de redes de ciudades (Agenda 21 para la Cultura, Interlocal, Federación Española de Municipios y Provincias, etc.) más que de ámbitos estrictamente académicos.

Lejos de ser la excepción, en la Argentina no existen trabajos especializados de base empírica sobre la materia. Es en este contexto donde la Universidad Nacional de Tres de Febrero impulsó el proyecto de investigación Políticas Culturales en los Gobiernos Locales que presentaremos a continuación.

\section{EL MARCO POLÍTICO INSTITUCIONAL}

La República Argentina ha adoptado para su gobierno la forma «representativa republicana federal» ${ }^{1}$. En tanto republicano, el gobierno se reparte entre tres poderes: ejecutivo, legislativo y judicial. Por su parte, del carácter federal se desprende la coexistencia de un gobierno central («federal») y de 23 provincias, a lo que hay que sumar la Ciudad Autónoma de Buenos Aires. A su vez, cada provincia establece hacia el interior de su territorio diferentes formas de gobiernos locales, prevaleciendo la forma de municipio. También en algunos casos se da la organización en departamentos, los cuales están integrados por más de un municipio, y en los casos de bajo número de habitantes los gobiernos locales tienen un rango menor al de municipio, adoptando, por ejemplo, la forma de comunas. En el total del país coexisten unos casi 3,000 gobiernos locales, de los cuales unos 2,000 revisten el carácter pleno de municipios, y los restantes formas menores. 
Siguiendo la consigna constitucional de republicanismo, los municipios poseen gobiernos tripartitos: un ejecutivo, que por lo común adopta la forma de intendencia, un legislativo (Honorable Concejo Deliberante) y un judicial (Tribunal Municipal de Faltas). La tradición histórica en la Argentina ha dado por resultado que tanto a nivel nacional como de las provincias y los municipios, el peso del ejecutivo sea mayor que el de los otros dos poderes.

La estructura institucional de una intendencia por lo común consta del intendente y una diversidad de secretarías, subsecretarías y direcciones.

Tal como sucediera en otras partes del mundo, hasta no hace mucho los gobiernos locales en la Argentina tenían funciones muy acotadas, como el ordenamiento del espacio público, la prestación de algunos servicios básicos y poco más. Las transformaciones del Estado que se dieron en la década de los noventa tuvieron por corolario un mayor empoderamiento de los gobiernos locales, en parte como resultado de una política desbocada de descentralización que no tenía por objeto el aumento de la capacidad del gobierno local sino la reducción del Estado central, y en parte por una revalorización de lo local cuyos orígenes habría que buscar, entre otros, en un reflujo de las fuerzas globalizadoras.

Como sea, lo cierto es que cuando el gobierno nacional, siguiendo los lineamientos del Consenso de Washington, se desentendía de su rol de actor principal del desarrollo delegando esta función en el mercado, los gobiernos locales emergían a una «mayoría de edad», como es habitual decir. Ya no sólo se encargaron de la limpieza de las calles y el alumbrado público, sino que cargaron sobre sí la responsabilidad de ser protagonistas de sus propios procesos de desarrollo, de proveer servicios de mayor alcance y complejidad, y de trasvasar sus fronteras y la tutoría provincial para entablar contactos con el mundo entero.

La denominada crisis de diciembre de 2001, que tras fuertes movilizaciones populares 
marcó el final del gobierno del entonces presidente Fernando de la Rúa, fue la bisagra en cuanto a la visión del rol del Estado en la Argentina. Desde entonces, y sobre todo desde la asunción de la presidencia de Néstor Kirchner en 2003, comienza un proceso que, si bien no puede ser caracterizado como de vuelta atrás, sí tiene un eje en la revalorización del papel del Estado como procurador del bien común. Un elemento que, sin embargo, marca una continuidad, es la valorización del ámbito local como espacio de desarrollo dada su condición de proximidad con la ciudadanía.

En el terreno de la gestión de políticas públicas en cultura, la Argentina tiene en la Secretaría de Cultura de la Nación, dependiente en forma directa del Ejecutivo nacional, la nave insignia de su política cultural. A la Secretaría se vinculan una serie de organismos descentralizados y, como tales, con un fuerte poder de autodeterminación respecto de aquélla, como sucede con el Instituto Nacional de Cine y Artes Audiovisuales, el Instituto Nacional del Teatro, la Comisión Nacional de Bibliotecas Populares, etc. Las provincias, por su parte, presentan disímiles soluciones institucionales para el desarrollo de sus políticas culturales. La mayoría cuentan con secretarías, otras con ministerios, y algunas con institutos, entes o formas similares. Decir qué sucede en los municipios en este sentido no resulta tarea sencilla dado que no existe un registro que dé cuenta de las agencias de cultura de los gobiernos locales de todo el país, y de hecho nos consta que no todos los gobiernos locales cuentan con un área dedicada a cultura. Cuando sí las tienen, sus rangos institucionales van desde subdirecciones hasta secretarías, como ya dijéramos, pero dada la falta de información confiable no podemos afirmar que no existan agencias que adopten otras formas.

Hasta aquí una breve descripción del contexto político institucional en que se desarrollan las políticas culturales en la Argentina y en el que se ha venido trabajando desde el proyecto de investigación Políticas Culturales a Nivel Local, el cual a continuación describiremos con mayor precisión antes de explayarnos sobre algunos de sus resultados. 


\section{EL PROYECTO. MARCO TEÓRICO Y METODOLÓGICO}

Las políticas culturales comienzan un proceso de reconocimiento en paridad con otras políticas públicas en la década los sesenta, fuertemente impulsado, entre otras instancias, por la UNESCO. En las décadas siguientes se impulsó un trabajo de desarrollo conceptual e investigativo en numerosos países, como bien describe Néstor García Canclini (1987). Hacia fines de los ochenta, los estudios sobre políticas culturales en Latinoamérica habían transitado de las descripciones burocráticas a la conceptualización crítica, de las cronologías y discursos a la investigación empírica, de las políticas gubernamentales a los movimientos sociales, y de los análisis nacionales a la investigación internacional. Por su parte, Juan Luis Mejía Arango (1999), en un artículo que se presenta como continuación del antes mencionado y que da cuenta de las transformaciones en el campo de las políticas culturales latinoamericanas en los últimos veinte años, pone de relieve un muy nutrido trabajo que, sobre todo desde el campo de los estudios culturales, múltiples intelectuales y centros de estudio vienen realizando en nuestro continente. Sin embargo, se pregunta el autor, «¿por qué motivo semejante producción intelectual no logra influir de manera contundente en las políticas?», poniendo de manifiesto un aparente divorcio entre el campo del pensamiento y el de la gestión pública en la materia.

La Argentina no ha estado ajena a este proceso descripto para el general del continente impulsado por García Canclini y Mejía, por los tempranos trabajos de Edwin Harvey (1990), fundamentalmente orientados al estudio de la institucionalidad y la legislación comparada, y por otros autores como Adolfo Colombres (1991) o Ezequiel Ander-Egg (1986, 1992), sin olvidar la fuerte impronta que en estas tierras ha tenido el catalán Tony Puig Picart (1994, 1995, 2000). La producción intelectual que en las últimas décadas sucediera a estos primeros nombres es prácticamente inabarcable: Ricardo Santillán Güemes y Héctor Olmos (2001, 2004) impulsan desde sus producciones una permanente reflexión sobre la práctica de la gestión cultural, Ana Wortman (2005, 2009) ha analizado las transformaciones recientes en el campo cultural, de los medios de comunicación y los consumos culturales, Octavio Getino $(1995,2006)$ ha generado una cuantiosa producción 
sobre las industrias culturales, Rosalía Winocur (1996), con un libro prácticamente desconocido, ha aportado uno de los más lúcidos trabajos de evaluación sobre la implementación de un programa cultural, y el recuento podría dilatarse ampliamente. Sin embargo, en este muy desarrollado estado del arte prácticamente no aparece el problema de las políticas culturales a nivel local ${ }^{2}$ dado que las iniciativas de los gobiernos locales en cultura no fueron objeto de reflexión por parte de la academia y menos aún de investigación empírica.

Es en este contexto, y en virtud de esta falencia, cuando en el año 2007 comienza a tomar forma en la Universidad Nacional de Tres de Febrero el equipo multidisciplinario de investigación que llevará adelante el proyecto Políticas Culturales a Nivel Local.

Si bien la escasa información previa sobre la materia obligó a que las primeras etapas fueran eminentemente exploratorias, desde un principio estaba claro que el objetivo del trabajo consistía en sondear los modelos de gestión puestos en acción por las agencias de cultura de los gobiernos locales, su factibilidad para el desarrollo de políticas culturales y su aporte a la capacidad estatal, entendida esta última como «la aptitud de las instancias de gobierno para plasmar, a través de políticas públicas, los máximos niveles posibles de valor social» (Repetto 2003).

Desde lo metodológico, el proyecto consiste básicamente en una serie de estudios de caso. Las primeras herramientas que se diseñaron para el trabajo fueron entrevistas en profundidad a los funcionarios, observaciones de los ámbitos de trabajo y análisis presupuestarios. Ya desde el principio se hizo evidente la necesidad de prestar oídos a los destinatarios de las políticas, por lo que se comenzó a aplicar el método de grupos focales y, por último, en el 2009 se implementó el trabajo etnográfico a través de la participación activa en las agencias estudiadas de miembros del equipo de investigación.

Hasta la fecha han sido estudiados doce municipios de la provincia de Buenos Aires, nueve 
pertenecientes al denominado Gran Buenos Aires ${ }^{3}$ (cinturón urbano que rodea a la ciudad de Buenos Aires) y tres en el interior de la provincia. ${ }^{4}$

\section{ALGUNOS RESULTADOS}

A continuación se presentan brevemente algunos aspectos que el trabajo de investigación ha develado sobre el quehacer cultural de los gobiernos municipales en la Argentina. Los distintos tópicos en que se organiza la presentación en modo alguno representan todas ellas ideas desarrolladas por el autor, sino que son el trabajo de los distintos miembros del equipo y del esfuerzo de reflexión conjunta.

DESARROLLO INSTITUCIONAL ${ }^{5}$

El primer elemento a destacar, y que debe servir de marco para lo que más abajo se comentará, es el hecho de que las políticas culturales van ganando espacio en la definición de las agendas de los gobiernos locales estudiados. Dos indicadores que apuntan en este sentido son: el desarrollo institucional y el avance en la participación presupuestaria que en los últimos años muestran los casos estudiados.

Aunque el tema presupuestario se abordará adelante con más detalle, digamos sin embargo que la estructura presupuestaria es un indicador reflejo de la agenda pública, es decir, de los temas que para un determinado gobierno han adquirido el rango de problema público.

Entre las agencias municipales de cultura estudiadas existen las que podríamos caracterizar como de muy larga data y que presentan el mismo rango institucional desde hace décadas, como por ejemplo la del municipio de Avellaneda. ${ }^{6}$ Otras, que constituyen la mayoría de los casos observados, han ido "ganando posiciones» en el organigrama del ejecutivo municipal. Un ejemplo de este tipo es el municipio de Moreno, ${ }^{7}$ que hasta el año 2006 incluía cultura simplemente como una mención dentro de una Coordinación General de 
Gobierno y Relaciones con la Comunidad, en la que se concentraban junto a cultura temas tan disímiles como defensa civil, comunicaciones, protocolo o juzgado de faltas. En el año 2007 se creó un programa en este municipio que tenía como únicos campos de trabajo relaciones institucionales y culturales, y finalmente en el 2008 se creó la Secretaría de Relaciones Institucionales, Culturales y Deportivas.

Aun en aquellas dependencias de cultura que, como mencionábamos antes, poseen un rango histórico, es posible detectar elementos que manifiestan un avance en la política cultural. Siguiendo por ejemplo con el caso de Avellaneda, se debe mencionar que sus institutos de formación artística, de reconocida excelencia académica más allá del distrito, recientemente han sido trasformados en universidades.

\section{LOS OBJETIVOS $^{8}$}

Si bien los objetivos de una política pública son el resultado de la disputa y negociación permanente entre los actores que intervienen en el campo en cuestión - funcionarios, estructura burocrática, normativas vigentes, grupos de influencia, opinión pública, etc.-, también es cierto que la sedimentación de experiencias, la tradición, el campo académico y otros factores impulsan la institucionalización de objetivos «básicos» que presumiblemente serían esperables de dicha política pública (Subirats 1989).

En el terreno de las políticas culturales podemos decir que entre los más difundidos de estos objetivos «básicos» institucionalizados se hallan la consolidación de valores nacionales, la difusión universal de las manifestaciones culturales a la ciudadanía, la promoción de la producción artística, la preservación del patrimonio y. más recientemente, el respeto por la diversidad cultural y el empleo de la cultura como un factor paliativo para problemas sociales no tradicionalmente vinculados al campo cultural. Así, hoy se entiende que desde las políticas culturales se puede aportar a la generación de empleo, a la inclusión social, al fortalecimiento de la ciudadanía, etc. 
En el marco de la investigación, la principal herramienta diseñada para captar los objetivos de las políticas culturales municipales es la entrevista en profundidad realizada a los funcionarios de primera línea. De lo que éstos manifiestan se desprende que participan del discurso generalizado respecto de lo que hemos denominado objetivos "básicos" que circula en el mundo occidental desde hace décadas. En el análisis tanto de los objetivos expresados por los directivos como de aquéllos implícitos en sus gestiones, Marcela Rebón (2011) ha identificado lo que denomina «tres grandes ejes» sobre los que se orientan las políticas culturales locales: social, político y económico. Entre los objetivos sociales son de destacar la inclusión social, la reducción de las desigualdades y la mejor distribución de los servicios culturales. En el eje político se pueden mencionar el fortalecimiento de los valores democráticos de la sociedad y la reducción de los niveles de conflictividad social. Por último, en el registro económico se trabaja en la generación de emprendimientos de base cultural, en la capacitación para la inserción en el mercado laboral y en el desarrollo de una oferta turístico cultural.

\section{LA NOMINACIÓN DEL SUJETO DESTINATARIO ${ }^{9}$}

A partir del cogito cartesiano, y con ello podríamos decir de la modernidad misma, la figura del sujeto se torna central en las formas sociales que caracterizamos como modernas. La forma en que el sujeto es nominado no es un factor menor en la constitución de éste. Históricamente, el sujeto en los sistemas políticos democráticos representativos lo constituía el ciudadano, el ciudadano era el soberano, por y para el que el sistema político desplegaba su dinámica. Como bien analiza Ignacio Lewkowicz (2004) para el caso argentino, las transformaciones ocurridas durante los 90 - siguiendo los vientos del Consenso de Washington-, instalaron la figura del consumidor como un nuevo sujeto del sistema político, ${ }^{10}$ «estamos ante el agotamiento práctico de un modelo de lazo social», dirá Lewkowicz. En el marco de la investigación, José Basualdo hizo una revisión analítica de las Constituciones argentinas $(1853,1949,1994)$ constatando, no sólo las afirmaciones de Lewkowicz, sino también la importancia que la cultura revestía en la malograda 
Constitución de 1949.

Teniendo en cuenta este antecedente histórico, José Tasat (2011) se pregunta quién es el actual sujeto de las políticas culturales municipales: «Las políticas públicas, se dirá, como curso de acción de los derechos o las demanda de la sociedad, construyen un destinatario, que es nombrado en sus atributos». Así, el destinatario de las políticas educativas es el alumno, de las políticas sociales, el beneficiario, o de las políticas económicas, empresarios y trabajadores. Por su parte, para las agencias de cultura municipales estudiadas el destinatario de sus políticas es «el asistente».

La nominación prefigura al sujeto, se constituye en institución y es instituyente, y modela los comportamientos de los actores intervinientes en la dinámica de la transacción cultural que el municipio propone: prestadores del servicio y beneficiarios están en alguna medida condicionados por ésta. En el campo educativo, por ejemplo, la histórica adopción del vocablo alumno, de origen griego, nos habla de una continuidad con la cultura grecolatina del mundo occidental. Por otra parte, alumno es el que se alimenta y que alimenta su espíritu a través de la enseñanza, siguiendo preceptos iluministas. El asistente, por su parte, es simplemente «el que concurre y está presente» en la eventual acción en que la cultura es puesta en acto. Esto tiene algunas implicancias interesantes: por un lado, y en el plano de la filosofía misma de la política cultural en juego, el asistente se acerca al fenómeno cultural pero no es parte de él. Por otra parte, y en términos de gestión, esto actúa como una limitante de las aspiraciones de resultado, dado que la sola asistencia da por cumplida la misión de la agencia de cultura; un conteo es todo el mecanismo de evaluación necesario, dejando fuera de foco el verdadero impacto que en términos socioculturales pudiera haber tenido la política.

\section{LA VOZ DE LOS «ASISTENTES»}

En el marco de la investigación, los grupos focales promovidos por Lorena Fiore constituyen los espacios donde la voz de los beneficiarios de las políticas culturales locales 
es escuchada. Dos son los resultados más relevantes del análisis de las entrevistas: el tránsito de una situación de agradecimiento conformista hacia una visión crítica y de demanda, y los imaginarios que los destinatarios construyen en relación a su apropiación de la oferta cultural.

La Argentina es un país con una larga tradición en derechos sociales, tal vez el más notorio de éstos es el derecho a la educación primaria pública, laica y obligatoria, sancionada por la ley 1420 de 1884. La ampliación y férrea internalización en la sociedad de los derechos sociales se dio durante los gobiernos peronistas (1946-1955). Posteriormente, la ola neoliberal, que en el país tuvo su germen en 1976 con la dictadura militar y fue decididamente abrazada en la década de los noventa, ocasionó una reingeniería social en la que los derechos sociales fueron uno de los factores a combatir. A pesar de ello, en el imaginario social pervivió la noción de derecho social, aunque el concepto se asocia más habitualmente a temas tales como educación, seguridad, salud, hábitat o espacios públicos que a cultura. Por lo general, la cultura es sólo considerada como un derecho por quienes de alguna forma participan del campo artístico-cultural, no así por el grueso de la ciudadanía.

En este marco, la primera reacción de los vecinos que, ajenos habitualmente a la cosa cultural, se acercan a la oferta de servicios que les ofrece el municipio, es de gratitud y tienden a magnificar el servicio. Un indicador de ello es que, ignorantes de la inversión de la comuna en los servicios de los que hacen usufructo, tienden a creer que ésta es mayor de lo que en realidad es. Sin embargo, quienes participan de los servicios rápidamente internalizan la noción de derecho cultural y, como corolario, el agradecimiento por un servicio no esperado se convierte en demanda, y la demanda no transita sólo por el eje prestación-no prestación, sino que se sumerge más profundamente para exigir calidad en el servicio. Llegada la instancia del reclamo, los aspectos más demandados son: mayor difusión de las propuestas, mayor carga horaria para las actividades y mayor nivel de profesionalización en la organización de los servicios. 
Como dijéramos anteriormente, otro de los resultados destacados en los grupos focales de beneficiarios es la forma en que las personas perciben en sus imaginarios los servicios culturales. Tras analizar múltiples entrevistas, hemos podido construir tres metáforas que responden, de manera global, a la visión que la mayoría de los participantes ${ }^{11}$ : el atelier, la proveeduría y la clínica.

En tanto atelier, los talleres son vistos como espacios de formación artística, de aprendizaje y de capacitación para poder acceder a alguna salida laboral; pero al promover la internalización de un canon estético hegemónico, en este sentido, y siguiendo a Miller y Yudice (2004), estos espacios actúan como formadores del gusto por excelencia. Como proveeduría, las agencias de cultura son el destino de muchos ciudadanos que, o bien buscan satisfacer necesidades y por tanto demandan servicios o, mayoritariamente son parte del campo artístico y ven en éstas una fuente de recursos que van desde espacios para exponer sus producciones hasta fondos para costear sus formaciones, pasando por recursos técnicos para sus espectáculos, etc. Por último, están aquellos ciudadanos que asisten en busca de un espacio terapéutico, sea por iniciativa propia o profesional. Las prácticas artísticas serían en este sentido un espacio para la distención, para contactar con elementos anímicos que la enajenación de la vida moderna mantiene acallados.

\section{LOS RECURSOS. NO TAN ESCASOS COMO SE SUPONÍA ${ }^{12}$}

El presupuesto constituye una herramienta para la definición de la agenda pública y, en función de las partidas asignadas, el gobierno establece la trascendencia que las distintas áreas tienen para su gestión.

El análisis de la situación presupuestaria en los municipios estudiados arroja resultados encontrados: por una parte, desde una perspectiva estrictamente cuantitativa la situación resulta muy alentadora; pero, por otra, la denominada «cultura del presupuesto» a que aspiran las sucesivas transformaciones del Estado, dista bastante de ser lo esperado en 
muchos distritos.

El análisis presupuestario se realizó con base en modelos del campo de las políticas culturales. Siguiendo esta línea, el principal objetivo del trabajo consistió en establecer los montos totales asignados a la agencia de cultura local, su participación del presupuesto total del distrito, el gasto por habitante y la evolución en el tiempo para series trienales de estas variables, y efectuando comparaciones con variables similares para el orden nacional y provincial.

Sobre la participación del total del presupuesto cabe una consideración previa. Existe un axioma basado en una supuesta recomendación de la UNESCO —recomendación que, hasta donde nuestro rastreo llegó, nunca logró formalizarse como tal — que exhorta a que los países miembros asignen el $1 \%$ del presupuesto a cultura. Esta consigna se ha convertido en un reclamo permanente del sector cultural. En Argentina, el presupuesto de la Secretaría de Cultura de la Nación está estacionado en el orden del $0.1 \%{ }^{13}$ y el de la provincia de Buenos Aires en el 0.35\%. Sin embargo, los municipios estudiados están todos, salvo alguna excepción y para algún período particular, por encima del 1\%, llegando este valor hasta el 4\%, lo cual también constituye una excepción.

En un contexto inflacionario del orden del 10\% anual para los indicadores oficiales (y el doble para los privados), obviamente los presupuestos públicos han ido creciendo significativamente de ejercicio en ejercicio, por lo que el gasto por habitante debe ser considerado año a año. Para el año 2007, tomado como ejemplo por ser uno de los que más datos consignan, la nación invertía 3.46 pesos/habitante (\$/h), la provincia 7.09 \$/h, en tanto que los municipios estudiados oscilaron entre 4.92 y $22.0 \$ / \mathrm{h}$.

La evolución en el tiempo de las variables indica, además, un incremento en el aporte destinado a cultura en los gobiernos locales, mientras que en la nación y en la provincia de Buenos Aires se encuentran estacionados en los porcentajes que mencionamos, $0.1 \% \mathrm{y}$ 
0.35\% respectivamente. Los municipios muestran entonces un avance en la participación de sus presupuestos, aunque es más difícil hacer una generalización para el comportamiento global de las variables que para su análisis individual. Digamos por ejemplo, que Florencio Varela ${ }^{14}$ avanzó desde el $1.28 \%$ en 2005 hasta un 2.61\% en 2008.

Sin embargo, el dato tal vez más inesperado lo representa el importante aporte en términos absolutos que los municipios realizan en relación a la nación o la provincia. En el año 2007, por ejemplo, la suma del presupuesto en cultura de ocho municipios representaba el 26.2\% del presupuesto nacional, y el 33.8\% del presupuesto provincial para el mismo lapso, lo cual rompe con un prejuicio completamente infundado según el cual los gobiernos locales no son significativos en términos de aporte financiero al sector cultural.

\section{CONCLUSIONES}

La situación de las agencias de cultura de los gobiernos locales en la Argentina presenta una oportunidad para la generación de políticas de desarrollo, inclusión y generación de lazos sociales de base cultural. Aunque el cuadro presenta algunas notorias deficiencias, el sostenido desarrollo de la institucionalidad en los últimos años hace pensar que los gobiernos locales son un terreno propicio para cambios reparadores en el área de la cultura.

Sin pretender ser exhaustivos, mencionaremos a continuación algunos de los factores que alimentan este diagnóstico. En primer lugar, debe destacarse el panorama alentador en términos de avance de la institucionalidad en cultura que ya hemos mencionado. Parte del mismo proceso, pero que amerita una mención especial, es en qué medida una parte muy significativa de los funcionarios de cultura actualmente en funciones participan de lo que podríamos llamar el discurso generalizado de las políticas culturales. Asumir esa base conceptual los hace entender la cultura tanto desde un registro antropológico como estético, y ser partícipes de ideas tales como el respeto por la diversidad, la democratización del acceso a los servicios culturales, el aliento de la participación universal en el desarrollo 
cultural, los derechos culturales, etc. No puede dejar de mencionarse como un factor positivo la base material con la que cuentan muchos municipios ${ }^{15}$, y no sólo en términos estrictamente presupuestarios, tal como hemos señalado más arriba, sino también en forma de infraestructuras como museos, auditorios, centros culturales, etc.

Entre los factores adversos no pueden dejar de considerarse los déficits en materia de herramientas de gestión de las agencias de cultura locales, los deficientes o inexistentes mecanismos de evaluación, el desconocimiento y las alteraciones de las estructuras presupuestarias, la falta de planificación, etc. Otro elemento a superar es la falta de comunicación con otros actores y el consecuentemente deficitario — cuando no ausentetrabajo asociativo. Las agencias de cultura de los municipios tienen un escaso diálogo con sus destinatarios, con instancias superiores (órganos de cultura provinciales y/o nacionales, de cuyas políticas a su vez son destinatarios), con otras agencias del propio municipio, con la sociedad civil e incluso con otras agencias de cultura de otros gobiernos locales. Por último, las áreas de cultura municipales están en deuda con la generación de propuestas creativas e innovadoras que vayan más allá de talleres de artes, el espectáculo o la preservación del patrimonio.

Para concluir, puede afirmarse que la generación de políticas públicas de cultura por parte de los gobiernos locales en la Argentina ofrece el panorama de un presente respetable y de un futuro alentador, pero para alcanzarlo habrá que transitar una senda de trabajo y transformación. 


\section{BIBLIOGRAFÍA}

Ander-Egg, E., 1992, Desarrollo y política cultural, Ciccus, Buenos Aires.

——, 1986, Metodología y práctica de la animación socio cultural, Humanitas, Buenos Aires.

Colombres, A., 1991, Manual del promotor cultural, Humanitas y Colihue, Buenos Aires.

García Canclini, N., (editor), 1987, Políticas culturales en América Latina, Grijalbo, México.

Getino, O., 2006, El capital de la cultura. Las industrias culturales en Argentina y en la integración Mercosur, Parlamento Cultural del Mercosur, Buenos Aires.

—, 1995, Las industrias culturales en la Argentina. Dimensión económica y políticas públicas, Colihue, Buenos Aires.

Harvey, E., 1990, Políticas culturales en Iberoamérica y el mundo, Tecnos, Madrid.

Lewkowicz, I., 2004, Pensar sin Estado. La subjetividad en la era de la fluidez, Paidós, Buenos Aires.

Mejía Arango, J. L., 1999, «Apuntes sobre políticas culturales en América Latina 19872009», en Revista Pensamiento Iberoamericano, n. 4, Segunda Época, Agencia Española de Cooperación para el Desarrollo, Madrid, pp. 105-129.

Miller, T. y G. Yúdice, 2004, Política cultural, Gedisa, Barcelona.

Puig Picart, T., 2000, Ciudad y cultura en el siglo XXI. Un paseo por el bosque de la 
gestión; 605 ideas y un método, Ciccus, Buenos Aires.

— 1995 , «La gestión de la ciudad ante el próximo milenio», en Nuevos paradigmas de la acción cultural. Agentes públicos, privados y sociales de AA.VV., Ciccus, Buenos Aires.

——, 1994, Animación sociocultural e integración territorial, Ciccus, Buenos Aires.

Rebón, M., 2011, «El estudio de la institucionalidad de las políticas culturales de los gobiernos locales», en Anuario de Indicadores Culturales 2010, UNTREF, Caseros.

Repetto, F., 2003, «Capacidad estatal: requisito necesario para una mejor política social en América Latina», ponencia presentada en el VIII Congreso Internacional del CLAD sobre la Reforma del Estado y de la Administración Pública, 28-31 de octubre, Panamá.

Santillán Güemes, R. y H. Olmos, (compiladores), 2004, El gestor cultural. Ideas y experiencias para su capacitación, Ciccus, Buenos Aires.

—_, (compiladores), 2001, Capacitar en cultura, Subsecretaría de Cultura de la Provincia de Buenos Aires, Buenos Aires.

Subirats, J., 1989, Análisis de políticas públicas y eficacia de la administración, INAP, Madrid.

Tasat, J. y P. Mendes Calado, 2009, «Indicadores presupuestarios: dispositivos para la gestión de los gobiernos locales», en Anuario de Indicadores Culturales 2009, UNTREF, en http://www.untref.edu.ar/documentos/indicadores\%202009/Indicadores\%20\%20Jose\%20Alejandro\%20Tasat.pdf

Tasat, J., 2011, «El destinatario de las políticas culturales de los gobiernos locales», en 
Anuario de Indicadores Culturales 2010, UNTREF, Caseros.

Winocur, R., 1996, De las políticas a los barrios. Programas culturales y participación popular, Miño y Dávila, Buenos Aires.

Wortman, A. (compiladora), 2009, Entre la política y la gestión de la cultura y el arte. Nuevos actores en la Argentina contemporánea, EUDEBA, Buenos Aires.

Wortman, A., 2005, «El desafío de las políticas culturales en la Argentina», en Cultura, política y sociedad, compilado por Matto, D., CLACSO, Buenos Aires.

\section{NOTAS}

${ }^{1}$ Conforme lo establece la Constitución Nacional en su artículo $1^{\circ}$.

${ }^{2}$ La excepción la constituyen La política Cultural a Nivel Local, de Ezequiel Ander-Egg, un muy breve y elemental trabajo de tipo manual; el mencionado trabajo de Rosalía Winocur, De las políticas a los barrios, que analiza la implementación de un programa de la entonces municipalidad de la Ciudad de Buenos Aires; y algunos breves trabajo sobre cultura y desarrollo local.

${ }^{3}$ Avellaneda, Berazategui, Florencio Varela, Moreno, Morón, San Fernando, Tigre, Tres de Febrero y Vicente López.

${ }_{5}^{4}$ General Pueyrredón, Olavarría y Pergamino.

5 Para ampliar este tema ver el trabajo de Rebón (2011), (próximamente en http://www.untref.edu.ar/indicadores_culturales.htm)

${ }^{6}$ Municipio industrial de la denominada «zona sur» del Conurbano Bonaerense que limita con la Ciudad de Buenos Aires.

${ }^{7}$ Municipio ubicado $40 \mathrm{~km}$ al oeste de la ciudad de Buenos Aires, perteneciente al denominado «tercer cordón del conurbano bonaerense», donde la mancha urbana se funde en el paisaje rural.

${ }^{8}$ Para ampliar este tema ver el trabajo de Rebón (2011).

9 Para ampliar el tema ver el trabajo de Tasat (2011), (próximamente en http://www.untref.edu.ar/indicadores_culturales.htm).

${ }^{10}$ En la Argentina la reforma constitucional de 1994 consagra en su artículo 42 los derechos del consumidor, incorporando al carácter constitucional un nuevo sujeto antes no contemplado: el consumidor.

${ }^{11}$ Cabe mencionar que los grupos focales se organizan mayoritariamente con asistentes a los talleres artísticos que brindan los municipios.

${ }_{12}$ Para ampliar el tema ver el trabajo de Tasat y Mendes (2009).

${ }^{13}$ Este porcentaje corresponde exclusivamente a la asignación de la mencionada Secretaría y no a la asignación de las dependencias descentralizadas. Según la forma de considerar la construcción de este indicador en distintos trabajos, este porcentaje puede llegar a duplicarse o casi triplicarse.

${ }^{14}$ Municipio ubicado $30 \mathrm{~km}$ al sur de la Ciudad de Buenos Aires.

${ }^{15}$ En modo alguno podemos generalizar esto para toda la Argentina, pero seguramente esto sí sucede con más casos de los que habitualmente se cree. 
Fecha de recepción: 23 de marzo de 2011.

Fecha de aceptación: 5 de octubre de 2011. 